\title{
Phase III trials examining the efficacy of cetirizine ophthalmic solution $0.24 \%$ compared to vehicle for the treatment of allergic conjunctivitis in the conjunctival allergen challenge model
}

This article was published in the following Dove Press journal:

Clinical Ophthalmology

\author{
Edward J Meier' \\ Gail L Torkildsen² \\ Paul J Gomes ${ }^{3}$ \\ Mark C Jasek ${ }^{4}$ \\ 'Apex Eye, Mason, OH, USA; ${ }^{2}$ Andover \\ Eye, Andover, MA, USA; ${ }^{3}$ Allergy, Ora, \\ Inc., Andover, MA, USA; ${ }^{4}$ Eyevance \\ Pharmaceuticals, TX, USA
}

Purpose: The purpose of these Phase III studies was to evaluate the efficacy and safety of cetirizine ophthalmic solution $0.24 \%$ compared with vehicle in the treatment of allergen-induced conjunctivitis using the Ora conjunctival allergen challenge $(\mathrm{CAC})^{\circledR}$ model.

Methods: The single-center (Study 1) and multi-center (Study 2), double-masked, randomized, vehicle-controlled, parallel group, CAC studies were conducted over $\sim 5$ weeks and four study visits. The study design only differed in entry criteria: Study 2 required more severe allergic conjunctivitis symptoms. Subjects were screened for an allergen response at Visits 1 and 2 and then randomized at Visit 3. Approximately 100 subjects were randomized in each study. The primary efficacy endpoints were ocular itching and conjunctival redness 15 minutes and 8 hours post-treatment, post-CAC.

Results: Cetirizine treatment administered 15 minutes or 8 hours prior to CAC resulted in significantly lower ocular itching at all time points post-CAC $(P<0.0001)$ compared to vehicle in both studies. Conjunctival redness measured by the investigator was significantly lower after cetirizine treatment compared to vehicle at 7 minutes post-CAC at both 15 minutes and 8 hours post-treatment in both studies $(P<0.05)$. All secondary endpoints were in favor and confirmatory of cetirizine efficacy with significant improvement in chemosis, eyelid swelling, tearing, ciliary redness, and episcleral redness, as well as nasal symptoms (rhinorrhea, nasal pruritus, ear or palatal pruritus, and nasal congestion) post-CAC. The most robust treatment differences were observed in Study 2 where more severe symptoms were required for study entry $(P<0.05)$. No safety concerns for cetirizine ophthalmic solution $0.24 \%$ were identified.

Conclusion: Cetirizine ophthalmic solution $0.24 \%$ was shown to be efficacious in the treatment of ocular and nasal signs and symptoms associated with allergic conjunctivitis and demonstrated a favorable safety profile. Clinical efficacy was demonstrated with a 15-minute onset of action and an $\sim 8$-hour duration of action.

Keywords: ocular allergy, cetirizine, ocular itching, safety, topical administration

\section{Introduction}

Allergic conjunctivitis affects $<15 \%$ of the global population including as much as $30 \%$ of the US population ${ }^{1}$ and up to $50 \%$ of the European population. ${ }^{2}$ Allergic conjunctivitis reactions range from a mild, self-limiting disease to a debilitating condition that significantly impairs one's quality of life. An individual's allergic response can be triggered by a variety of stimuli, including tree and grass pollens, animal hair and dander, and other environmental insults. This response manifests in a cascade of ocular
Correspondence: Mark C Jasek Eyevance Pharmaceuticals, 777 Taylor Street, Suite 1050, Fort Worth, TX 76102, USA

Tel +I 8176776127

Email mjasek@eyevance.com 
symptoms including itching, redness, chemosis, tearing, and eyelid swelling.

The signs and symptoms of allergic conjunctivitis progress through an early acute phase and a late phase in response to an allergen. Allergens interact with $\operatorname{IgE}$ bound to sensitized mast cells activating enhanced levels of histamine and causing degranulation. ${ }^{3}$ In the acute phase, release of histamine and other proallergic mediators induces itching, vasodilation, and vascular leakage, which lead to ocular redness, chemosis, and lid swelling. Mast cells synthesize and release cytokines, chemokines, and growth factors that initiate a cascade of inflammatory events leading to a final late phase reaction characterized by the recruitment of eosinophils, neutrophils, and macrophages in the conjunctival tissues. ${ }^{4,5}$

Measuring allergic conjunctivitis in a controlled environment has been difficult in the past due to hypervariability dependent on allergen, subject, season, and weather, ${ }^{6}$ resulting in different symptomatic responses and a range of severity. ${ }^{7}$ The Ora conjunctival allergen challenge $(\mathrm{CAC})^{\circledR}$ model circumvents these concerns inducing a moderate-to-severe allergic reaction in a controlled and reproducible manner. ${ }^{8}$ In the CAC model, all subjects undergo a screening procedure where subjects demonstrate reproducible moderate-to-severe allergic responses. This model is an established method approved by regulatory agencies to determine therapeutic efficacy in the relief of allergic signs and symptoms. Numerous studies have demonstrated the clinical efficacy of therapeutics for the indication of allergic conjunctivitis using the CAC model including antihistamines, alcaftadine ${ }^{9}$ and olopatadine hydrochloride, ${ }^{10,11}$ and coritcosteroids such as Dextenza. ${ }^{12}$ Furthermore, bepotastine besilate ophthalmic solution was shown to be efficacious in the treatment of nasal symptoms using the CAC model, ${ }^{13}$ demonstrating a broad role for the $\mathrm{CAC}$ model in the development of allergic conjunctivitis therapeutics. As a result, the last several products approved for this indication (Pazeo [Alcon Pharmaceuticals; Fort Worth, TX, USA], Lastacaft [Allergan; Dublin, Ireland], Bepreve [Bausch \& Lomb; Rochester, NY, USA], and Pataday [Alcon Pharmaceuticals; Fort Worth, TX, USA]) have established efficacy through the CAC model.

Most of the approved treatments for ocular allergies are antihistamines and mast cell stabilizers, or both, and act to reduce the signs and symptoms of the early-phase reaction., ${ }^{74}$ Cetirizine hydrochloride $\left(Z^{2} y r t e{ }^{\circledR}\right.$; Johnson \& Johnson Consumer Inc; New Brunswick, NJ, USA) is the number one oral antihistamine allergy treatment recommended by allergists. ${ }^{15}$ Cetirizine ophthalmic solution $0.24 \%$ used in these studies was a sterile, buffered, clear, colorless aqueous solution containing cetirizine $0.24 \%$ (equivalent to cetirizine hydrochloride $0.29 \%$ ) and $0.010 \%$ benzalkonium chloride (preservative). ${ }^{16}$ Cetirizine hydrochloride selectively inhibits H1 histamine receptors to treat seasonal and perennial allergic rhinitis, idiopathic urticaria, and itching due to chronic hives ${ }^{17}$ and has a long-standing record of approval in different formulations. ${ }^{18}$ Cetirizine hydrochloride is safe and well tolerated and was approved in tablet form (5 and $10 \mathrm{mg}$ ) in USA in 1995, as a syrup formulation in 1996, for children aged 2-5 years in 1998, for children aged 6 months and older in 2002, and for nonprescription use as a chewable tablet and syrup in 2007. The long-standing therapeutic success and safety of cetirizine hydrochloride in different formulations and pronounced ocular symptoms of allergic conjunctivitis ${ }^{1}$ supported the development of cetirizine hydrochloride as an ophthalmic solution to combat the ocular effects of allergic conjunctivitis.

In 2018, cetirizine ophthalmic solution $0.24 \%$ (Zerviate $^{\mathrm{TM}}$ ) was approved by the Food and Drug Agency for the treatment of ocular itch associated with allergic conjunctivitis. Here, we report two Phase III efficacy studies that were the basis of this approval. These two studies show strong and similar anti-itch efficacy of cetirizine ophthalmic solution $0.24 \%$ compared with vehicle using the CAC model. Study 2 expanded the efficacy assessment in Study 1 to subjects with more severe allergic conjunctivitis symptoms including eyelid swelling. Together these studies show reproducible success of cetirizine ophthalmic solution $0.24 \%$ for the treatment of ocular and nasal signs and symptoms associated with allergic conjunctivitis in diverse patient populations. Clinical efficacy was demonstrated with a 15-minute onset of action and an $\sim 8$-hour duration of action.

\section{Methods}

\section{Study design}

Study 1 (single center) and Study 2 (multi-center) were double-masked, randomized, vehicle-controlled, parallel group, CAC studies. Both studies were performed in compliance with the Declaration of Helsinki, International Conference of Harmonization, Good Clinical Practice Guidance and all applicable local, state, and federal requirements. Each subject or their legally acceptable representative provided written informed consent prior to any study-related procedures. The study protocols, informed consent forms, and related documents were approved by the Investigational Review Board (IRB), Alpha IRB (San Clemente, CA, USA).

Inclusion and exclusion criteria included the following: subjects had to be $\geq 10$ years of age, provide written informed consent and assent if applicable, and sign the HIPAA form. Subjects must not have worn contact lenses for at least 
72 hours prior to and during the study trial period, had no known contraindications or sensitivities to the use of any of the study medication or the vehicle component, not have been pregnant, planning a pregnancy, or lactating, must not have had ocular surgical intervention within 3 months prior to or during the study and/or a history of refractive surgery within the past 6 months, must not have had a known history of retinal detachment, diabetic retinopathy, or progressive retinal disease, must not have had the presence of an active ocular infection (bacterial, viral, or fungal) or a positive history of an ocular herpetic infection, or preauricular lymphadenopathy at Visits 1, 2, and 3A, must not have had a history of status asthmaticus, a known history of persistent moderate or severe asthma, or a known history of moderate-to-severe allergic asthmatic reactions to any of the study allergens, and must have been able and willing to avoid all disallowed medications for the appropriate washout period and during the study.

At Visit 1, screening, subjects must have had a positive bilateral CAC reaction (greater than or equal to a score of 2 itching and greater than or equal to a score of 2 conjunctival redness) within 10 minutes of instillation of the last titration of allergen and, at Visit 2, had a positive bilateral CAC reaction (as above) for at least two of the three time points. Subjects must not have manifested signs or symptoms of clinically active allergic conjunctivitis in either eye at the start of Visits 1, 2, and 3A (defined as the presence of any itching or $>1$ redness in any vessel bed). Subjects were required to have a calculated best-corrected visual acuity of 0.7 logarithm of the minimum angle of resolution ( $\log \mathrm{MAR})$ or better in each eye as measured using an Early Treatment Diabetic Retinopathy Study (ETDRS) chart.

All subjects in Study 2 met the criteria above and the following criteria. Subjects must have had an average of greater than 2.5 itching and greater than 0.5 eyelid swelling for both eyes after post-CAC assessments at Visit 2. The requirement of ocular itching score and eyelid swelling was used to define Study 2 as "severe" allergic conjunctivitis for the purpose of this report.

\section{Treatments}

The investigational product, cetirizine ophthalmic solution $0.24 \%$, and its vehicle (formulation without cetirizine) were manufactured by Akorn, Inc. (Somerset, NJ, USA) and supplied in identical $10 \mathrm{~mL}$ sterile bottles. For masking purposes, bottles of active treatment and vehicle were identical in appearance. Each bottle had an identical label bearing information that met applicable regulatory requirements, as well as a subject number. Study treatment was packaged and labeled based on the randomization schedule generated prior to the start of the study.
At Visit 1 (Days -21 \pm 3 ), subjects' responses to the CAC and eligibility for trial participation were determined as described in the "Subject Criteria" section of each clinical study report. Any subject who failed to test positively to an allergen at Visit 1 was to be excluded from the study. At Visit 2 (Days $-14 \pm 3$ ), subjects received a confirmatory CAC, and ocular and nasal signs and symptoms were assessed to confirm an allergic response. At Visit 3A (Day 0), qualified subjects who satisfied the inclusion and exclusion criteria were enrolled and randomized evenly by site into one of the two treatment arms (active or vehicle) and received their first dose of study medication in each eye.

The primary efficacy comparisons of the mean differences between cetirizine ophthalmic solution $0.24 \%$-treated eyes and vehicle-treated eyes for ocular itching and conjunctival redness were assessed 8 hours (Visit 3B, Day 0) and 15 minutes (Visit 4, Day 14) after dosing with either study drug or vehicle. Analyses were done at 3, 5, and 7 minutes post-CAC for ocular itching and at 7, 15, and 20 minutes post-CAC for conjunctival redness and all secondary efficacy endpoints. The secondary efficacy measures were assessments of ciliary and episcleral redness, chemosis, eyelid swelling, tearing, nasal allergy symptoms (rhinorrhea, nasal pruritus, ear or palatal pruritus, nasal congestion), and a composite score of nasal symptoms. Ocular itching, conjunctival redness, ciliary and episcleral redness, chemosis, eyelid swelling, tearing, rhinorrhea, nasal pruritus, ear or palatal pruritus, and nasal congestion were assessed on a 5-point scale, where $0=$ none, $1=$ mild, $2=$ moderate, $3=$ moderate $/$ severe, and $4=$ severe, prior to and after the CAC. An outline of study design is provided in Figure 1.

Safety and tolerability are reported for three additional trials in Malhotra et al (2018, co-submitted). Tolerability measurements included drop comfort and descriptor at Visit 3A. Safety assessments included adverse events (AEs), visual acuity, slit lamp biomicroscopy, and dilated fundoscopy. All AEs were coded to system organ class and preferred term using MedDRA Version 16.1.

\section{Statistical methods}

The intent-to-treat (ITT) population was analyzed as randomized, and these data were used for all efficacy analyses. Primary efficacy analyses were conducted on the ITT population with last observation carried forward for missing data. Each variable was analyzed using an analysis of covariance (ANCOVA) model for each post-CAC time point for Visits $3 \mathrm{~B}$ and 4 with the average of the subjects' baseline post-CAC scores at Visit 2 as a covariate. Two-sample $t$-tests were used as unadjusted sensitivity analyses at each post-CAC 


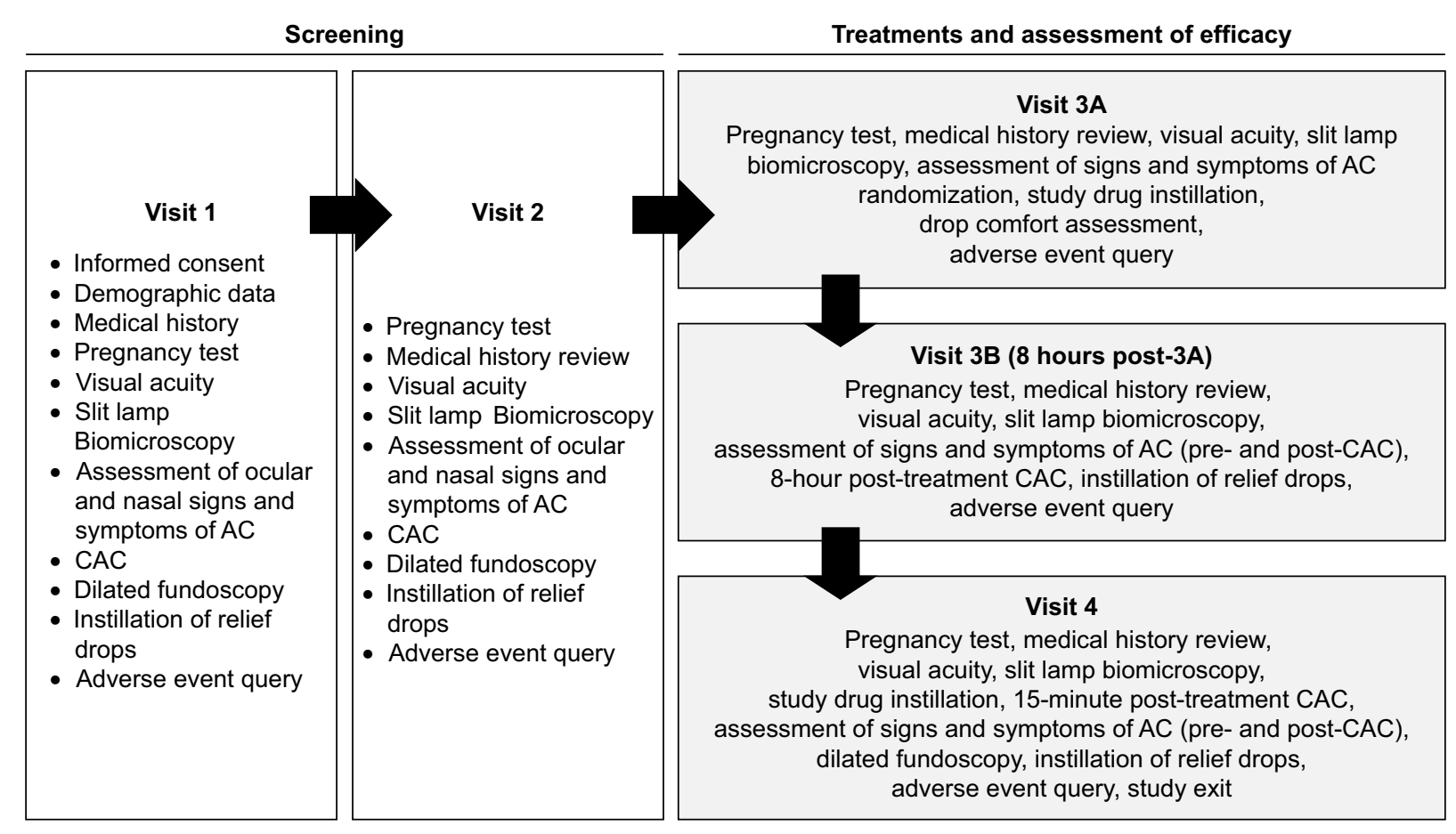

Figure I Study design.

Notes: During the four study visits, evaluations and treatments proceeded as follows: Visit I (Day $-2 I \pm 3$ ), screening procedures at Visit I determined subject eligibility; Visit 2 (Days -14 \pm 3 ), baseline procedures at Visit 2 confirmed subject eligibility; Visit 3 (Day 0), Visit 3 was divided into two office visits on the same day, such as Visit $3 \mathrm{~A}$ and Visit 3B. During Visit 3A, eligible subjects who had satisfied the inclusion and exclusion criteria were enrolled into the study, randomized, and received their first dose of either cetirizine ophthalmic solution $0.24 \%$ or vehicle ( $1-2$ drops) bilaterally. Visit $3 B$ assessed ocular and nasal signs and symptoms before and after the CAC. Visit 4 (Days 14 \pm 3 ): subjects received their second dose of either cetirizine ophthalmic solution $0.24 \%$ or vehicle (I-2 drops). Fifteen minutes after instillation, subjects underwent the bilateral CAC and ocular and nasal signs and symptoms were assessed.

Abbreviations: AC, allergic conjunctivitis; CAC, conjunctival allergen challenge.

time point as well as nonparametric Wilcoxon rank sum tests. At each post-CAC time point, treatment differences were considered statistically significant for each primary endpoint if they showed significance at a two-sided level of $\alpha=0.05$. Sensitivity or supportive analyses were performed on the ITT population with a multiple imputation method using the Markov Chain Monte Carlo, baseline observation carried forward and with observed data only.

The secondary efficacy variables (ciliary and episcleral redness, chemosis, eyelid swelling, tearing, rhinorrhea, nasal pruritus, ear or palatal pruritus, nasal congestion, and a composite score of presence or absence of at least one nasal symptom) were measured at Visits $3 \mathrm{~B}$ and 4 and were analyzed in a similar manner to primary efficacy variables on the ITT population with observed data only. Qualitative measures were analyzed using Fisher's exact tests or Chi-square tests as appropriate. The statistical model or test used for each variable is defined in the caption for its respective figure or table.

\section{Results}

\section{Subject disposition and demographics}

In Study 1, 100 subjects were enrolled and randomized into one of the two treatment arms at Visit 3A. Fifty subjects were assigned to each the cetirizine ophthalmic solution $0.24 \%$ group and the vehicle group. The 100 randomized subjects received their first dose of assigned study medication in-office at Visit 3A and comprise the ITT population. In Study 2, a total of 101 subjects were randomized into the ITT population (51 cetirizine ophthalmic solution $0.24 \%$ and $50 \%$ vehicles). The majority of subjects in each study completed all visits. In Study 1, 96\% of subjects completed the study (Table 1). In Study 2, 86.1\% of subjects completed the study. Subject demographics were well balanced across groups and similar in both studies (Table 2).

Ocular itching was assessed by subjects in each eye using a 5-point scale after treatment instillation, prior to and 3, 5, and 7 minutes after CAC. Efficacy of cetirizine ophthalmic solution $0.24 \%$ was assessed 15 minutes and 8 hours post-treatment instillation by comparing cetirizine ophthalmic solution $0.24 \%$ treatment scores to vehicle at each time point. Lower scores indicate reduced itching. In Study 1, baseline (pre-CAC) mean scores for both treatment groups were 0 , with a mean increase of more than $2.6 \mathrm{U}$ following $\mathrm{CAC}$, thus demonstrating successful induction of allergic conjunctivitis symptoms.

Cetirizine ophthalmic solution $0.24 \%$ was effective at preventing ocular itching at 15 minutes post-treatment instillation 
Table I Subject disposition

\begin{tabular}{|c|c|c|c|c|}
\hline & \multicolumn{2}{|c|}{ Study I: moderate allergic conjunctivitis } & \multicolumn{2}{|c|}{ Study 2: severe allergic conjunctivitis } \\
\hline & Cetirizine & Vehicle & Cetirizine & Vehicle \\
\hline Randomized & 50 & 50 & 51 & 50 \\
\hline PP population & $49(98.0 \%)$ & $47(94.0 \%)$ & $43(84.3 \%)$ & $44(88.0 \%)$ \\
\hline ITT population & $50(100.0 \%)$ & $50(100.0 \%)$ & $51(100.0 \%)$ & $50(100.0 \%)$ \\
\hline ITT subjects who completed the study & $49.0(98.0 \%)$ & 47 (94.0\%) & $43(84.3 \%)$ & $44(88.0 \%)$ \\
\hline \multicolumn{5}{|l|}{ Primary reason for withdrawal } \\
\hline Protocol violation & $0(0.0 \%)$ & $0(0.0 \%)$ & $3(5.9 \%)$ & $2(4.0 \%)$ \\
\hline Administrative reasons & $0(0.0 \%)$ & $\mathrm{I}(2.0 \%)$ & $3(5.9 \%)$ & $2(4.0 \%)$ \\
\hline $\begin{array}{l}\text { Manifest clinically active signs or symptoms } \\
\text { of allergic conjunctivitis at Visit } 4\end{array}$ & $0(0.0 \%)$ & $\mathrm{I}(2.0 \%)$ & $2(3.9 \%)$ & $2(4.0 \%)$ \\
\hline Adverse event & I $(2.0 \%)$ & I $(2.0 \%)$ & $0(0.0 \%)$ & $0(0.0 \%)$ \\
\hline
\end{tabular}

Notes: The number of subjects enrolled in each study and those in each population are shown. Percentages are based on the total number of subjects randomized in each treatment group.

Abbreviations: ITT, intent to treat; PP, per protocol.

(Figure 2 and Table 3). Post-CAC mean treatment differences were in favor of cetirizine ophthalmic solution $0.24 \%$ and were significantly lower than vehicle at 3,5 , and 7 minutes for both studies 15 minutes after treatment instillation $(P<0.0001$ for each time point) (Table 3 ). The cetirizine ophthalmic solution $0.24 \%$ treatment group had mean ocular itching score of at least $1 \mathrm{U}$ lower than the vehicle treatment 15 minutes after study medication instillation at 3,5 , and 7 minutes post-CAC The mean ocular itching score in the cetirizine ophthalmic solution $0.24 \%$ group was 1.0 compared to a mean score of 2.38 in the vehicle group, 15 minutes after treatment instillation and 3 minutes post-CAC, in Study 1 (moderate AC, Figure 2).

Study 2 results and treatment differences were confirmatory of strong ocular itching efficacy at 15 minutes

Table 2 Demographics

\begin{tabular}{|c|c|c|c|c|}
\hline & \multicolumn{2}{|c|}{ Study I: moderate allergic conjunctivitis } & \multicolumn{2}{|c|}{ Study 2: severe allergic conjunctivitis } \\
\hline & $\begin{array}{l}\text { Cetirizine } \\
n=50\end{array}$ & $\begin{array}{l}\text { Vehicle } \\
n=50\end{array}$ & $\begin{array}{l}\text { Cetirizine } \\
n=5 \text { I }\end{array}$ & $\begin{array}{l}\text { Vehicle } \\
n=50\end{array}$ \\
\hline Age (years), mean (SD) & $39.5(17.32)$ & $38.1(14.56)$ & $40.6(12.8)$ & $39.2(10.84)$ \\
\hline \multicolumn{5}{|l|}{ Gender } \\
\hline Male & $13(26.0 \%)$ & $20(40.0 \%)$ & $12(23.5 \%)$ & $20(40.0 \%)$ \\
\hline Female & $37(74.0 \%)$ & $30(60.0 \%)$ & 39 (76.5\%) & $30(60.0 \%)$ \\
\hline \multicolumn{5}{|l|}{ Ethnicity } \\
\hline Hispanic or Latino & II (22.0\%) & $4(8.0 \%)$ & $\mathrm{I}(2.0 \%)$ & $0(0.0 \%)$ \\
\hline Non-Hispanic or Latino & $39(78.0 \%)$ & $46(92.0 \%)$ & $50(98.0 \%)$ & $50(100.0 \%)$ \\
\hline \multicolumn{5}{|l|}{ Race } \\
\hline American Indian or Alaskan Native & $2(4.0 \%)$ & $0(0.0 \%)$ & $0(0.0 \%)$ & $0(0.0 \%)$ \\
\hline Asian & I (2.0\%) & $2(4.0 \%)$ & $0(0.0 \%)$ & $\mathrm{I}(2.0 \%)$ \\
\hline Black or African American & $3(6.0 \%)$ & $2(4.0 \%)$ & $10(19.6 .0 \%)$ & $17(34.0 \%)$ \\
\hline White & $44(88.0 \%)$ & $46(92.0 \%)$ & $4 \mathrm{I}(80.4 \%)$ & $31(62.0 \%)$ \\
\hline Others & $0(0.0 \%)$ & $0(0.0 \%)$ & $0(0.0 \%)$ & I (2.0\%) \\
\hline \multicolumn{5}{|l|}{ Iris color (per eye) } \\
\hline Black & $2(2.0 \%)$ & $0(0.0 \%)$ & $2(2.0 \%)$ & $4(4.0 \%)$ \\
\hline Blue & $34(34.0 \%)$ & $36(36.0 \%)$ & $36(35.3 \%)$ & $18(18.0 \%)$ \\
\hline Brown & $38(38.0 \%)$ & $36(36.0 \%)$ & $38(37.3 \%)$ & $60(60.0 \%)$ \\
\hline Hazel & $14(14.0 \%)$ & $14(14.0 \%)$ & $12(11.8 \%)$ & $6(6.0 \%)$ \\
\hline Green & 12 (I2.0\%) & $14(14.0 \%)$ & I4 (I3.7\%) & $12(12.0 \%)$ \\
\hline
\end{tabular}

Notes: The demographic information (age, gender, ethnicity, race, and iris color) of the ITT population is shown. Percentages are based on the total number of subjects in each treatment group except for iris color, which are based on the total number of eyes in each treatment group.

Abbreviation: ITT, intent to treat. 


\section{A}

Moderate AC

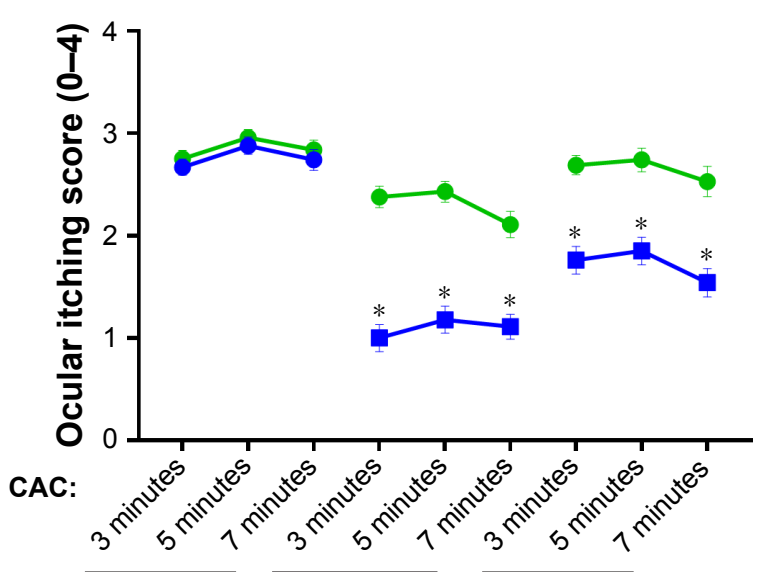

PT: Baseline 15 minutes 8 hours
B

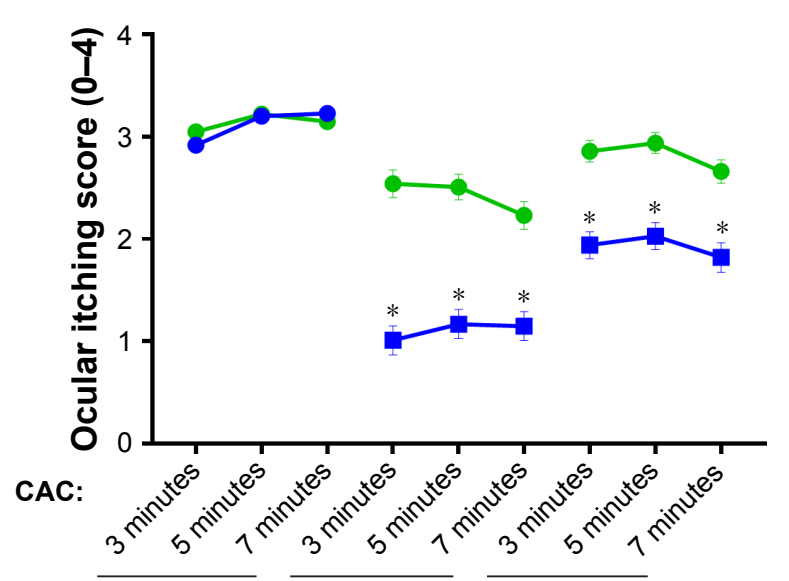

PT: Baseline 15 minutes 8 hours

\section{Cetirizine $\rightarrow-$ Vehicle}

Figure 2 Ocular itching.

Notes: Mean scores of the cetirizine ophthalmic solution $0.24 \%$ (blue) and the vehicle (green) group are shown from each study. Data shown are mean and standard error of the mean for each time point (3, 5, or 7 minutes post-CAC) after treatment ( 15 minutes or 8 hours postdosing). (A) Study I, $n=50$ in each treatment group. (B) Study 2 , $\mathrm{n}=5 \mathrm{I}$ and 50 in the cetirizine and vehicle treatments, respectively. $* \mathrm{P}<0.05$.

Abbreviations: AC, allergic conjunctivitis; CAC, conjunctival allergen challenge; PT, post-treatment.

post-treatment instillation. The greatest difference observed between cetirizine $0.24 \%$ ophthalmic solution treatment group and vehicle treatment group was $1.53 \mathrm{U}$ at 3 minutes post-CAC. Here, the mean ocular itching score was 2.54 in the vehicle group compared to 1.01 in the cetirizine ophthalmic solution $0.24 \%$ group (Figure 2 and Table 3). These studies demonstrate that cetirizine consistently provides rapid protection from ocular itching caused

Table 3 Change in ocular itching and conjunctival redness

\begin{tabular}{|c|c|c|c|c|}
\hline \multirow[t]{2}{*}{ Assessment } & \multicolumn{2}{|c|}{ Study I: moderate allergic conjunctivitis } & \multicolumn{2}{|c|}{ Study 2: severe allergic conjunctivitis } \\
\hline & $\Delta$ mean score & $P$-value & $\Delta$ mean score & $P$-value \\
\hline \multicolumn{5}{|c|}{ Ocular itching 15 minutes post-treatment } \\
\hline Post-CAC: 3 minutes & -1.38 & $<0.0001$ & -1.53 & $<0.0001$ \\
\hline Post-CAC: 5 minutes & -1.25 & $<0.0001$ & -1.34 & $<0.0001$ \\
\hline Post-CAC: 7 minutes & -1.00 & $<0.0001$ & -1.07 & $<0.0001$ \\
\hline \multicolumn{5}{|c|}{ Ocular itching 8 hours post-treatment } \\
\hline Post-CAC: 3 minutes & -0.93 & $<0.0001$ & -0.92 & $<0.0001$ \\
\hline Post-CAC: 5 minutes & -0.89 & $<0.0001$ & -0.90 & $<0.0001$ \\
\hline Post-CAC: 7 minutes & -0.99 & $<0.0001$ & -0.84 & $<0.0001$ \\
\hline \multicolumn{5}{|c|}{ Conjunctival redness 15 minutes post-treatment } \\
\hline Post-CAC: 3 minutes & -0.33 & 0.0006 & -0.46 & 0.0069 \\
\hline Post-CAC: 5 minutes & -0.15 & 0.1708 & -0.18 & 0.5763 \\
\hline Post-CAC: 7 minutes & -0.14 & 0.3281 & -0.25 & 0.2600 \\
\hline \multicolumn{5}{|c|}{ Conjunctival redness 8 hours post-treatment } \\
\hline Post-CAC: 3 minutes & -0.30 & 0.0259 & -0.42 & 0.0129 \\
\hline Post-CAC: 5 minutes & -0.03 & 0.9551 & -0.24 & 0.2924 \\
\hline Post-CAC: 7 minutes & -0.01 & 0.8790 & -0.31 & 0.1327 \\
\hline
\end{tabular}

Notes: Ocular itching and conjunctival redness were the primary endpoints for the symptoms and signs of allergic conjunctivitis, respectively. Ocular itching and conjunctival redness measurements were assessed on a 5-point scale described in the "Methods" section. Data shown are from the ITT population with last observation carried forward to account for missing data. P-value was calculated using an ANCOVA model with treatment and baseline (Visit 2) score in the model and comparing cetirizine ophthalmic solution $0.24 \%$ group with vehicle group.

Abbreviations: ANCOVA, analysis of covariance; CAC, conjunctival allergen challenge; ITT, intent to treat. 
by allergen exposure, regardless of response severity, just 15 minutes post-treatment instillation.

Cetirizine ophthalmic solution $0.24 \%$ demonstrated persistent efficacy at 8 hours post-treatment instillation (Figure 2 and Table 3). The cetirizine ophthalmic solution $0.24 \%$ group had significantly lower mean itching scores than vehicle treatment as early as 3 minutes post-CAC $(P<0.0001)$. The difference between the cetirizine ophthalmic solution $0.24 \%$ group and vehicle was near $1 \mathrm{U}$ and significantly different at all 8 hours post-treatment instillation post-CAC time points $(P<0.0001$ for each time point). The minimum difference between the groups was $-0.84 \mathrm{U}$ (Study 2, 7 minutes post-CAC), and the maximum difference in itching score was from $-0.99 \mathrm{U}$ (Study 1, 7 minutes post-CAC) compared to vehicle (Table 3). In Study 1, the mean ocular itching score at 7 minutes post-CAC was 1.54 in the cetirizine ophthalmic solution $0.24 \%$ group compared to 2.53 in the vehicle group. In Study 2, the mean ocular itching score at 7 minutes post-CAC was 1.82 in the cetirizine $0.24 \%$ ophthalmic solution group compared to 2.66 in the vehicle group. These studies demonstrate strong prolonged efficacy in the relief of ocular itching up to 8 hours after the instillation of cetirizine $0.24 \%$ ophthalmic solution.

Primary efficacy was further assessed by conjunctival redness measured by the investigator using a 5-point scale ( $0-4$, half units allowed) at 15 minutes and 8 hours post-treatment, as described in Figure 1, and at 7, 15, and
20 minutes post-CAC. Lower scores indicate less conjunctival redness. Baseline (pre-CAC) mean scores for both treatment groups were 0.28 (vehicle) and 0.34 (cetirizine) in Study 1 and 0.62 (vehicle) and 0.55 (cetirizine) in Study 2, consistent with the selection for more severe allergic conjunctivitis symptoms in Study 2. The mean conjunctival redness score increased in each treatment group increased to at least 2.38 in Study 1 and 2.62 in Study 2 following CAC demonstrating the induction of allergic conjunctivitis symptoms.

Treatment differences were in favor of the cetirizine ophthalmic solution $0.24 \%$ group compared to vehicle at all post-CAC time points 15 minutes and 8 hours posttreatment instillation. The mean conjunctival redness score was significantly lower at 7 minutes post-CAC in both studies ( $P=0.0006$ in Study 1 and $P=0.0069$ in Study 2, Table 3). Post-CAC scores at 15 and 20 minutes were consistently lower in both studies in the cetirizine ophthalmic solution $0.24 \%$ group compared to vehicle albeit, not statistically significant. These results suggest that treatment with cetirizine improves both signs and symptoms of $\mathrm{AC}$ in the majority of patients.

\section{Conjunctival and eyelid swelling}

Chemosis (swelling of the conjunctiva) and eyelid swelling scores were significantly improved in both studies (Table 4 and Figure 3). The investigator assessed these endpoints prior to $\mathrm{CAC}$ and then again at 7, 15, and 20 minutes post-CAC

Table 4 Change in secondary ocular efficacy endpoints post-treatment, post-CAC, all time points

\begin{tabular}{|c|c|c|c|c|}
\hline \multirow[t]{2}{*}{ Assessment } & \multicolumn{2}{|c|}{ Study I: moderate allergic conjunctivitis } & \multicolumn{2}{|c|}{ Study 2: severe allergic conjunctivitis } \\
\hline & $\Delta$ mean score & $P$-value & $\Delta$ mean score & $P$-value \\
\hline \multicolumn{5}{|l|}{ Chemosis (conjunctival swelling) } \\
\hline 15 minutes post-treatment & -0.38 & $<0.0001$ & -0.17 & 0.0478 \\
\hline 8 hours post-treatment & -0.32 & 0.0070 & -0.26 & 0.0087 \\
\hline \multicolumn{5}{|l|}{ Eyelid swelling } \\
\hline 15 minutes post-treatment & -0.30 & 0.0207 & -0.60 & $<0.0001$ \\
\hline 8 hours post-treatment & -0.40 & 0.0022 & -0.50 & $<0.0001$ \\
\hline \multicolumn{5}{|l|}{ Tearing } \\
\hline 15 minutes post-treatment & -0.30 & 0.0191 & -0.50 & 0.0004 \\
\hline 8 hours post-treatment & -0.10 & 0.3876 & -0.30 & 0.0278 \\
\hline \multicolumn{5}{|l|}{ Ciliary redness } \\
\hline I5 minutes post-treatment & -0.26 & 0.0007 & -0.30 & 0.0337 \\
\hline 8 hours post-treatment & -0.20 & 0.0719 & -0.20 & 0.0873 \\
\hline \multicolumn{5}{|l|}{ Episcleral redness } \\
\hline I5 minutes post-treatment & -0.16 & 0.0338 & -0.14 & 0.2105 \\
\hline 8 hours post-treatment & -0.15 & 0.1077 & -0.16 & 0.0911 \\
\hline
\end{tabular}

Notes: Data shown are pooled mean change from vehicle of all time points post-CAC (7, 15, and 20 minutes) from the ITT population with observation data only for secondary ocular efficacy endpoints: chemosis, tearing, eyelid swelling, ciliary redness, and episcleral redness. $P$-value calculated using a repeated measures ANCOVA model with treatment, time point, and baseline (visit two post-CAC average) score in the model and comparing cetirizine ophthalmic solution $0.24 \%$ group to vehicle group.

Abbreviations: ANCOVA, analysis of covariance; CAC, conjunctival allergen challenge; ITT, intent to treat. 


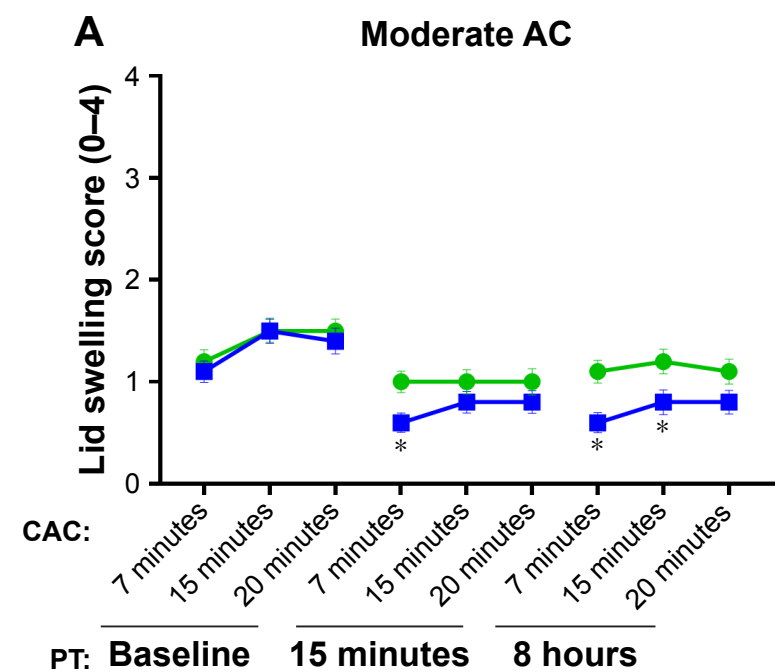

B

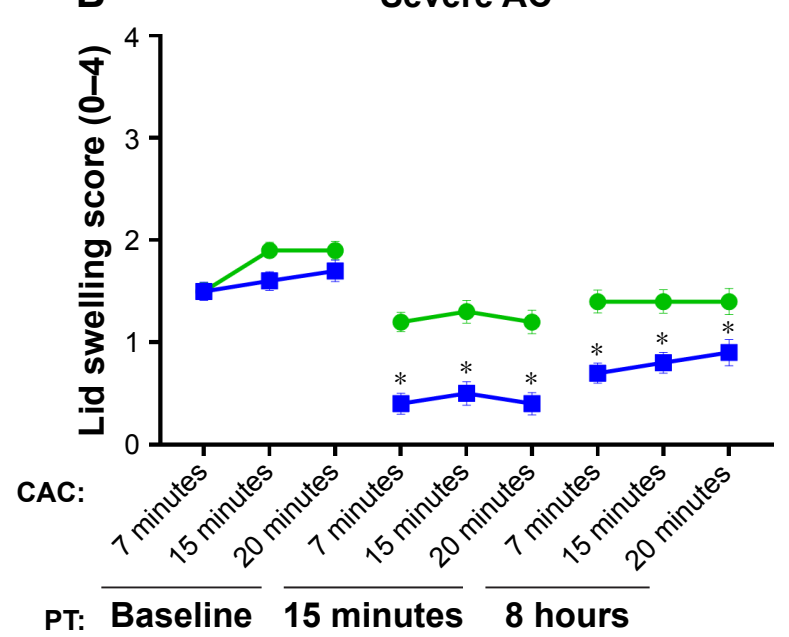

Cetirizine $\rightarrow-$ Vehicle

Figure 3 Eyelid swelling.

Notes: Mean scores of the cetirizine ophthalmic solution $0.24 \%$ (blue) and the vehicle (green) group are shown from each study. Data shown are mean and standard error of the mean for each time point (7, 15, or 20 minutes post-CAC) after treatment ( 15 minutes or 8 hours postdosing). (A) Study I, $n=50$ in each treatment group. (B) Study 2 , $\mathrm{n}=5 \mathrm{I}$ and 50 in the cetirizine and vehicle treatments, respectively. $* \mathrm{P}<0.05$.

Abbreviations: AC, allergic conjunctivitis; PT, post-treatment; $C A C$, conjunctival allergen challenge.

using a 5-point scale. Lower scores indicate clinical improvement. Chemosis was significantly lower across all post-CAC time points, at both 15 minutes and 8 hours post-treatment in both Study 1 and Study $2(P<0.05$ across all post-CAC time points) ranging from a difference of $0.17-0.38$ (Table 4). Eyelid swelling was also significantly improved across all post-CAC time points at both 15 minutes and 8 hours post-treatment, in both studies $(P<0.05$ in Study 1 and $P<0.0001$ in Study 2, Table 4). In Study 1, a mean difference of $0.30 \mathrm{U}$ at 15 minutes post-treatment and $0.40 \mathrm{U}$ at 8 hours post-treatment was observed. More severe eyelid swelling criteria were required for in Study 2 where a mean difference between treatment groups of $0.60 \mathrm{U}$ at 15 minutes post-treatment and $0.50 \mathrm{U}$ at 8 hours post-treatment was observed (Table 4). Greater differences between vehicle and cetirizine treatment were observed in Study 2, where swelling criteria were enriched for (through entry inclusion criteria) (Figure 3). These studies show that, in an enriched or non-enriched population, swelling is significantly improved by administering cetirizine ophthalmic solution $0.24 \%$ as preventative care up to 8 hours before allergen exposure.

Additional measurements were taken to assess the impact of cetirizine ophthalmic solution $0.24 \%$ treatment on less dominant signs and symptoms of allergic conjunctivitis. These secondary measurements include tearing, ciliary redness, and episcleral redness. Data for secondary endpoints were assessed using the ITT population with observed data only. All ocular signs and symptoms showed a trend of improvement in favor of cetirizine ophthalmic solution $0.24 \%$ treatment.

Significant improvement was observed in subject tearing. Subjects reported tearing pre-CAC and then again at 7, 15, and 20 minutes post-CAC both 15 minutes and 8 hours posttreatment. Tearing was significantly lower at 15 minutes posttreatment with cetirizine in Study $1(0.30 \mathrm{U}, P=0.0191)$ and Study 2 ( $0.50 \mathrm{U}, P=0.0004)$ across all post-CAC time points. The success of cetirizine treatment continued to be observed 8 hours post-treatment. In Study 2, a significant difference of $0.30(P=0.0278)$ was observed between the vehicle and cetirizine ophthalmic solution $0.24 \%$ treatment group. Tearing was not enriched for in this study; however, a trend toward a less in tearing in the active treatment group was observed across all time points, post-CAC, and post-treatment.

Ciliary and episcleral redness were also improved by treatment with cetirizine ophthalmic solution $0.24 \%$. Both ciliary and episcleral redness were evaluated by the investigator at 7,15 , and 20 minutes post-CAC at 15 minutes and 8 hours post-treatment. Lower scores indicate less redness and thus clinical improvement. Across all 15 minutes post-treatment post-CAC time points, the mean ciliary redness score was significantly lower in the cetirizine group compared to vehicle by $0.26 \mathrm{U}$ (Study 1, $P=0.0007$ ) and $0.30 \mathrm{U}$ (Study 2, $P=0.0337$ ) (Table 4). Eight hours post-treatment instillation, cilliary redness continued to be lower in the cetirizine treatment group 
across all post-CAC time points in both studies (Table 4). Similar success was observed in episcleral redness such that mean redness scores were consistently lower in the cetirizine ophthalmic solution $0.24 \%$ group compared to the vehicle group (Table 4). In Study 1, episcleral redness was significantly $0.16 \mathrm{U}$ lower in the cetirizine $0.24 \%$ ophthalmic solution treatment group compared to the vehicle group $(P=0.0338)$. Similar differences were observed at 8 hours post-treatment instillation and in Study 2 (Table 4).

Allergic conjunctivitis symptoms also manifest through nasal symptoms that are activated by histamine receptors. ${ }^{19}$ Rhinorrhea, nasal pruritus, ear or palatal pruritus, and nasal congestion were measured by subjects on a 5-point scale where lower scores indicate improvement. The mean score for every nasal symptom was in favor of the cetirizine ophthalmic solution $0.24 \%$ group across all three time points (Table 5). The most notable superiority in the cetirizine group was rhinorrhea. Rhinorrhea was significantly lower by $0.4-0.7 \mathrm{U}$ across all post-CAC time points, at both 15 minutes post-treatment and 8 hours post-treatment $(P<0.05$, Table 5).

Significant improvement was further observed in all other measured nasal symptoms. Nasal pruritus was significantly improved across all post-CAC time points at 8 hours post-treatment in Study $1(P=0.0184)$ and 15 minutes post-treatment in Study $2(P=0.0043)$ (Table 5). Ear or palatal pruritus was significantly improved in Study 2, where the mean score was significantly lower in the cetirizine ophthalmic solution $0.24 \%$ group than in the vehicle by
$0.5 \mathrm{U}(P=0.0025)$ at 15 minutes post-treatment and $0.6 \mathrm{U}$ $(P<0.0001)$ at 8 hours post-treatment. Nasal congestion was significantly improved in the cetirizine ophthalmic solution $0.24 \%$ group compared to vehicle across all treatment groups at 8 hours post-treatment $(-0.4 \mathrm{U}, P=0.0209)$ in Study 1 and at both 15 minutes post-treatment $(-0.7 \mathrm{U}, P=0.0003)$ and 8 hours post-treatment $(-0.4 \mathrm{U}, P=0.0020)$ in Study 2 (Table 5). These studies show the success of cetirizine $0.24 \%$ ophthalmic solution at improving nasal signs and symptoms of allergic conjunctivitis.

Significant improvement was further observed in all other measured nasal symptoms. Nasal pruritus was significantly improved across all post-CAC time points at 8 hours post-treatment in Study $1(P=0.0184)$ and 15 minutes post-treatment in Study $2(P=0.0043)$ (Table 5). Ear or palatal pruritus was significantly improved in Study 2, where the mean score was significantly lower in the cetirizine ophthalmic solution $0.24 \%$ group than in the vehicle by $0.5 \mathrm{U}(P=0.0025)$ at 15 minutes post-treatment and $0.6 \mathrm{U}(P<0.0001)$ at 8 hours post-treatment. Nasal congestion was significantly improved in the cetirizine ophthalmic solution $0.24 \%$ group compared to vehicle across all treatment groups at 8 hours post-treatment $(-0.4 \mathrm{U}, P=0.0209)$ in Study 1 and at both 15 minutes post-treatment $(-0.7 \mathrm{U}, P=0.0003)$ and 8 hours post-treatment $(-0.4 \mathrm{U}, P=0.0020)$ in Study 2 (Table 5).

To further assess the effect of cetirizine on nasal signs and symptoms of allergic conjunctivitis, a binary composite nasal score was calculated to assess an overall change in allergic conjunctivitis nasal symptoms. The presence of any

Table 5 Change in nasal symptoms post-treatment, post-CAC, all time points

\begin{tabular}{|c|c|c|c|c|}
\hline \multirow[t]{2}{*}{ Assessment } & \multicolumn{2}{|c|}{ Study I: moderate allergic conjunctivitis } & \multicolumn{2}{|c|}{ Study 2: severe allergic conjunctivitis } \\
\hline & $\Delta$ mean score & $P$-value & $\Delta$ mean score & $P$-value \\
\hline \multicolumn{5}{|l|}{ Rhinorrhea } \\
\hline 15 minutes post-treatment & -0.4 & 0.0080 & -0.7 & 0.0001 \\
\hline 8 hours post-treatment & -0.7 & $<0.0001$ & -0.5 & 0.0030 \\
\hline \multicolumn{5}{|l|}{ Nasal pruritus } \\
\hline 15 minutes post-treatment & -0.1 & 0.5429 & -0.5 & 0.0043 \\
\hline 8 hours post-treatment & -0.3 & 0.0184 & -0.3 & 0.0536 \\
\hline \multicolumn{5}{|l|}{ Ear or palatal pruritus } \\
\hline 15 minutes post-treatment & -0.3 & 0.0548 & -0.5 & 0.0025 \\
\hline 8 hours post-treatment & -0.2 & 0.1412 & -0.6 & $<0.0001$ \\
\hline \multicolumn{5}{|l|}{ Nasal congestion } \\
\hline 15 minutes post-treatment & -0.2 & 0.2614 & -0.7 & 0.0003 \\
\hline 8 hours post-treatment & -0.4 & 0.0209 & -0.4 & 0.0020 \\
\hline
\end{tabular}

Notes: Data shown are mean change from vehicle of all time points post-CAC (7, 15, and 20 minutes) from the ITT population with observation data only for nasal symptoms of allergic conjunctivitis: rhinorrhea, nasal pruritus, ear or palatal pruritus, and nasal congestion. $P$-value calculated using a repeated measures ANCOVA model with treatment, time point, and baseline (Visit 2 post-CAC average) score in the model and comparing cetirizine ophthalmic solution $0.24 \%$ group with vehicle group. Abbreviation: ANCOVA, analysis of covariance; CAC, conjunctival allergen challenge; ITT, intent to treat. 
Table 6 Proportion of subjects with nasal symptoms

\begin{tabular}{|c|c|c|c|c|}
\hline \multirow[t]{2}{*}{ Assessment } & \multicolumn{2}{|c|}{ Study I: moderate allergic conjunctivitis } & \multicolumn{2}{|c|}{ Study 2: severe allergic conjunctivitis } \\
\hline & $\begin{array}{l}\text { Cetirizine } \\
\mathrm{n}=50\end{array}$ & $\begin{array}{l}\text { Vehicle } \\
n=50\end{array}$ & $\begin{array}{l}\text { Cetirizine } \\
n=51\end{array}$ & $\begin{array}{l}\text { Vehicle } \\
\mathrm{n}=\mathbf{5 0}\end{array}$ \\
\hline \multicolumn{5}{|l|}{15 minutes post-treatment } \\
\hline Pre-CAC & $9(18.4 \%)$ & $10(20.4 \%)$ & $6(13.3 \%)$ & $5(11.1 \%)$ \\
\hline Post-CAC: 7 minutes & $32(65.3 \%) *$ & $39(83.0 \%)$ & $22(51.2 \%)^{*}$ & $36(81.8 \%)$ \\
\hline Post-CAC: 15 minutes & $40(81.6 \%)$ & 43 (9l.5\%) & $25(58.1 \%)^{*}$ & $38(86.4 \%)$ \\
\hline Post-CAC: 20 minutes & $38(77.6 \%) *$ & $44(93.6 \%)$ & $31(72.1 \%)$ & $39(88.6 \%)$ \\
\hline \multicolumn{5}{|l|}{8 hours post-treatment } \\
\hline Pre-CAC & 7 (I4.3\%) & $6(12.0 \%)$ & $3(6.0 \%)$ & $\mathrm{I}(2.1 \%)$ \\
\hline Post-CAC: 7 minutes & $30(61.2 \%)^{*}$ & $41(82.0 \%)$ & $29(58.0 \%)^{*}$ & $42(87.5 \%)$ \\
\hline Post-CAC: 15 minutes & $40(81.6 \%)$ & $47(94.0 \%)$ & $39(78.0 \%)$ & $43(89.6 \%)$ \\
\hline Post-CAC: 20 minutes & $4 \mathrm{I}(83.7 \%)$ & 47 (94.0\%) & $42(84.0 \%)$ & $43(89.6 \%)$ \\
\hline
\end{tabular}

Notes: The composite score is calculated based on the presence or absence of at least one nasal symptom. *In the cetirizine column, the $P$-value is $<0.05$ (exact $P$-values are in the corresponding text). P-values were calculated using a Fisher's exact test, comparing cetirizine ophthalmic solution $0.24 \%$ group with the vehicle group. Abbreviation: CAC, conjunctival allergen challenge.

one of the above measured nasal symptoms would score as "present" for the outcome of proportion of subjects with any nasal symptoms. Significant improvement was observed as early as 15 minutes post-treatment at the first post-CAC time point ( 7 minutes) where cetirizine ophthalmic solution $0.24 \%$ caused a significantly lower proportion of subjects to develop nasal symptoms than vehicle in Study $2(P=0.0031)$ (Table 6). The corresponding time point in Study 1 was also improved, albeit not statistically significant $(P=0.0635)$. This trend continued at later post-CAC time points (15 and 20 minutes) and was statistically significant at 20 minutes post-CAC in Study $1(P=0.0407)$ and 15 minutes post-CAC in Study $2(P=0.0040)$ (Table 6). At 8 hours post-treatment, a significant reduction in the proportion of subjects with nasal symptoms was seen in Study $1(P=0.0266)$ and Study $2(P=0.0014)$ at 7 minutes post-CAC. Consistent with the 15 minutes post-treatment measurements, all other time points were also in favor of cetirizine ophthalmic solution $0.24 \%$ treatment (Table 6). These studies show success of cetirizine $0.24 \%$ ophthalmic solution at improving nasal signs and symptoms of allergic conjunctivitis.

\section{Safety}

There were no safety concerns in either study. There were no serious AE or severe AEs reported in either study. Only four subjects reported ocular treatment-emergent AEs in Study 1, of which only one AE (mild eye pain) was reported in the cetirizine group that was suspected to be related to the study treatment. Similar AE reporting frequency was observed in both the vehicle and cetirizine groups in Study 1. There were no ocular or nonocular treatment-emergent AEs that were suspected to be related to the study treatment in Study 2 . Favorable drop comfort scores with a mean discomfort score of below 3 on a scale of $0-10$, where 0 is the most comfortable and 10 is the least comfortable, were seen in both studies (Table 7). No clinically significant shifts or safety concerns were identified by dilated fundoscopy, slit lamp biomicroscopy, or visual acuity assessments in any study.

\section{Discussion}

These two studies form the basis of Food and Drug Administration approval of Zerviate (cetirizine ophthalmic solution $0.24 \%$ ), the newest topical antiallergic for the treatment of

Table 7 Drop comfort assessments

\begin{tabular}{|c|c|c|c|c|}
\hline \multirow[t]{2}{*}{ Assessment } & \multicolumn{2}{|c|}{ Study I: moderate allergic conjunctivitis } & \multicolumn{2}{|c|}{ Study 2: severe allergic conjunctivitis } \\
\hline & $\begin{array}{l}\text { Cetirizine } \\
n=50\end{array}$ & $\begin{array}{l}\text { Vehicle } \\
n=50\end{array}$ & $\begin{array}{l}\text { Cetirizine } \\
n=5 \text { I }\end{array}$ & $\begin{array}{l}\text { Vehicle } \\
n=50\end{array}$ \\
\hline \multicolumn{5}{|l|}{ Drop comfort, mean (SD) } \\
\hline Upon installation & $1.2(1.42)$ & $1.0(1.49)$ & $1.6(2.02)$ & $0.8(1.22)$ \\
\hline I minute postinstallation & $1.4(1.44)$ & $1.2(1.60)$ & $3.0(2.33)$ & I.I (I.I4) \\
\hline 2 minutes postinstallation & $\mathrm{I} .3(\mathrm{I} .44)$ & $1.0(1.50)$ & $2.6(2.14)$ & I.I (I.I3) \\
\hline
\end{tabular}

Notes: Drop comfort was measured on a scale of $0-10$, where 0 was the most comfortable and 10 was the least comfortable. Analysis was performed using the safety populations in both studies. 
ocular itching. Ocular itching is the most disruptive symptom reported by subjects of allergic conjunctivitis. ${ }^{7}$ Currently, oral/systemic antiallergics are inefficient at treating ocular symptoms of an allergic response $\mathrm{e}^{20,21}$ and require hours for the onset of action. ${ }^{15}$ Cetirizine ophthalmic solution $0.24 \%$ demonstrated consistent superiority to vehicle for ocular itching in both studies, a rapid onset of action and a duration of action similar to other ophthalmic solutions. ${ }^{22-24}$ Thus, cetirizine ophthalmic solution $0.24 \%$ has the potential to quickly address the needs of a patient whose ocular symptoms are not being met by an oral therapeutic.

Coupled with the strong efficacy, cetirizine ophthalmic solution offers a unique advantage to patients as an ophthalmic solution. Allergic conjunctivitis encompasses a range of diseases, and thus, the ideal therapeutic for one patient will not ease the symptoms of all patients emphasizing the need for a broad market of therapeutics for this indication. ${ }^{6}$ Cetirizine has been administered as an oral medication for over 23 years familiarizing many patients and physicians with how well this drug works for them as an individual and its side effects. As an ophthalmic solution, the side effects of cetirizine are further diminished, most notably, cetirizine ophthalmic solution $0.24 \%$ did not cause drowsiness in the studies reported here nor in two studies with the enrollment of over 500 subjects each (Malhotra et al, 2018, co-submitted). The safety profile of cetirizine ophthalmic solution $0.24 \%$ was favorable and consistent in all studies. Thus, cetirizine ophthalmic solution $0.24 \%$ is a familiar therapeutic with few side effects and the potential to significantly reduce ocular itching due to allergic conjunctivitis.

An advantage of the CAC model is the ability to measure the individual signs and symptoms of allergic conjunctivitis. In addition to ocular itching, cetirizine ophthalmic solution $0.24 \%$ had extended efficacy toward the improvement of other ocular signs and symptoms of allergic conjunctivitis including conjunctival redness, chemosis, eyelid swelling, ciliary redness, and episcleral redness even in the absence of any enrichment (Study 1). Cetirizine ophthalmic solution $0.24 \%$ provided some relief for conjunctival redness, similar to other approved therapeutics, ${ }^{14}$ although the pathophysiology of redness is not directly targeted by antihistamines. ${ }^{25}$ For all other ocular efficacy endpoints, the cetirizine ophthalmic solution $0.24 \%$ group was significantly improved compared with vehicle for at least one time point and frequently for several time points. Most notably, cetirizine $0.24 \%$ ophthalmic solution had a robust impact on eyelid and conjunctival swelling (chemosis), which was even more pronounced in an enriched population (Study 2, where swelling was entry criteria). These two studies corroborate the success of cetirizine ophthalmic solution $0.24 \%$ for all standard signs and symptoms of allergic conjunctivitis.

Furthermore, cetirizine demonstrated efficacy in the reduction of multiple nasal symptoms even when provided as a topical ophthalmic solution. Rhinorrhea, nasal pruritus, ear or palatal pruritus, and nasal congestion were significantly improved compared to vehicle at one or more post-CAC time points. Improvements in scores were most robust for the symptom of rhinorrhea where all post-CAC measurements were significantly improved from vehicle and nasal pruritus where the majority of time points were significantly improved from vehicle. Thus, cetirizine ophthalmic solution $0.24 \%$ may further help address the needs of a patient who has rhinoconjunctivitis whose nasal symptoms are not being met by an oral therapeutic.

\section{Conclusion}

Cetirizine ophthalmic solution $0.24 \%$ is a strong therapeutic, with an ability to provide quick and extended relief of ocular and nasal symptoms of allergic conjunctivitis. There are no safety concerns identified with cetirizine treatment and drop comfort ranged from very comfortable to comfortable. As an ophthalmic solution, cetirizine offers a faster onset of action for ocular and nasal signs and symptoms than the oral formulation and is aided by patient and physician familiarity.

\section{Acknowledgment}

Medical writing assistance was provided by Ora, Inc.

\section{Disclosure}

Paul Gomes is an employee of Ora, Inc. Mark C Jasek is an employee of Eyevance Pharmaceuticals. Zerviate is a proprietary trademark of Nicox SA, and is licensed by Eyevance Pharmaceuticals. Ora Conjunctival Allergen Challegen $\left(\right.$ Ora-CAC $\left.{ }^{\circledR}\right)$ is a registered trademark of Ora, Inc. The other authors report no conflicts of interest in this work.

\section{References}

1. Bielory L. Allergic and immunologic disorders of the eye. Part II: ocular allergy. J Allergy Clin Immunol. 2000;106(6):1019-1032.

2. Burbach GJ, Heinzerling LM, Röhnelt C, et al. Ragweed sensitization in Europe - GA(2)LEN study suggests increasing prevalence. Allergy. 2009;64(4):664-665

3. La Rosa M, Lionetti E, Reibaldi M, et al. Allergic conjunctivitis: a comprehensive review of the literature. Ital J Pediatr. 2013;39:18-18.

4. Bacon AS, Ahluwalia P, Irani AM, et al. Tear and conjunctival changes during the allergen-induced early- and late-phase responses. J Allergy Clin Immunol. 2000;106(5):948-954.

5. Ciprandi G, Buscaglia S, Pesce GP, Bagnasco M, Canonica GW. Ocular challenge and hyperresponsiveness to histamine in patients with allergic conjunctivitis. J Allergy Clin Immunol. 1993;91(6):1227-1230. 
6. Ehlers WH, Donshik PC. Allergic ocular disorders: a spectrum of diseases. Clao J. 1992;18(2):117-124.

7. Gomes PJ. Trends in prevalence and treatment of ocular allergy. Curr Opin Allergy Clin Immunol. 2014;14(5):451-456.

8. Abelson MB, Chambers WA, Smith LM. Conjunctival allergen challenge. A clinical approach to studying allergic conjunctivitis. Arch Ophthalmol. 1990;108(1):84-88.

9. Nakatani H. Alcaftadine $0.25 \%$ versus olopatadine $0.1 \%$ in preventing cedar pollen allergic conjunctivitis in Japan. A Randomized Study. 2018.

10. Mclaurin E, Narvekar A, Gomes P, Adewale A, Torkildsen G. Phase 3 randomized double-masked study of efficacy and safety of once-daily $0.77 \%$ olopatadine hydrochloride ophthalmic solution in subjects with allergic conjunctivitis using the conjunctival allergen challenge model. Cornea. 2015;34(10):1245-1251.

11. Mclaurin E, Bergmann M, Narvekar A, Adewale A, Gomes PJ, Torkildsen G. Pooled analysis of two studies evaluating efficacy and safety of olopatadine hydrochloride $0.77 \%$ in patients with allergic conjunctivitis. Clinical Ophthalmology (Auckland, NZ.). 2017;11:1089-1097.

12. Torkildsen G, Abelson MB, Gomes PJ, Mclaurin E, Potts SL, Mah FS. vehicle-controlled, Phase 2 clinical trial of a sustained-release dexamethasone intracanalicular insert in a chronic allergen challenge model. J Ocul Pharmacol Ther. 2017;33(2):79-90.

13. Cavet ME, Gomes PJ, Carr WW, Williams JI. Bepotastine besilate ophthalmic solution $1.5 \%$ for alleviating nasal symptoms in patients with allergic conjunctivitis. J Asthma Allergy. 2018;11:29-39.

14. Abelson MB, Shetty S, Korchak M, Butrus SI, Smith LM. Advances in pharmacotherapy for allergic conjunctivitis. Expert Opin Pharmacother. 2015;16(8):1219-1231.

15. Johnson \& Johnson Consumer Companies, I. Zyrtec The \#1 Allergist Recommended Brand*, Available from: http://www.multivu.com/ assets/52078/documents/Zyrtec-fact-sheet-99-original.pdf. 2011.

16. FDA. Perscribing Information for Zerviate TM, Available from: https:// www.accessdata.fda.gov/drugsatfda_docs/label/2017/208694s0001bl. pdf. 2017.
17. Zhang L, Cheng L, Hong J. The clinical use of cetirizine in the treatment of allergic rhinitis. Pharmacology. 2013;92(1-2):14-25.

18. Portnoy JM, Dinakar C. Review of cetirizine hydrochloride for the treatment of allergic disorders. Expert Opin Pharmacother. 2004;5(1): $125-135$.

19. White MV. The role of histamine in allergic diseases. J Allergy Clin Immunol. 1990;86(4 Pt 2):599-605.

20. Lanier BQ, Abelson MB, Berger WE, et al. Comparison of the efficacy of combined fluticasone propionate and olopatadine versus combined fluticasone propionate and fexofenadine for the treatment of allergic rhinoconjunctivitis induced by conjunctival allergen challenge. Clin Ther. 2002;24(7):1161-1174

21. Spangler DL, Abelson MB, Ober A, Gotnes PJ. Randomized, doublemasked comparison of olopatadine ophthalmic solution, mometasone furoate monohydrate nasal spray, and fexofenadine hydrochloride tablets using the conjunctival and nasal allergen challenge models. Clin Ther. 2003;25(8):2245-2267.

22. Nakatani H, Gomes P, Bradford R, Guo Q, Safyan E, Hollander DA. Alcaftadine $0.25 \%$ versus Olopatadine $0.1 \%$ in preventing cedar pollen allergic conjunctivitis in Japan: a randomized study. Ocul Immunol Inflamm. 2018:1-10.

23. Mclaurin EB, Marsico NP, Ackerman SL, et al. Ocular itch relief with alcaftadine $0.25 \%$ versus olopatadine $0.2 \%$ in allergic conjunctivitis: pooled analysis of two multicenter randomized clinical trials. Adv Ther. 2014;31(10):1059-1071.

24. Ackerman S, D'Ambrosio F, Greiner JV, Villanueva L, Ciolino JB, Hollander DA. A multicenter evaluation of the efficacy and duration of action of alcaftadine $0.25 \%$ and olopatadine $0.2 \%$ in the conjunctival allergen challenge model. $J$ Asthma Allergy. 2013;6:43-52.

25. Ono SJ, Abelson MB. Allergic conjunctivitis: update on pathophysiology and prospects for future treatment. J Allergy Clin Immunol. 2005; 115(1):118-122.
Clinical Ophthalmology

\section{Publish your work in this journal}

Clinical Ophthalmology is an international, peer-reviewed journal covering all subspecialties within ophthalmology. Key topics include: Optometry; Visual science; Pharmacology and drug therapy in eye diseases; Basic Sciences; Primary and Secondary eye care; Patient Safety and Quality of Care Improvements. This journal is indexed on Submit your manuscript here: http://www.dovepress.com/clinical-ophthalmology-journal

\section{Dovepress}

PubMed Central and CAS, and is the official journal of The Society of Clinical Ophthalmology (SCO). The manuscript management system is completely online and includes a very quick and fair peer-review system, which is all easy to use. Visit http://www.dovepress.com/ testimonials.php to read real quotes from published authors. 